\title{
Ranking of fuzzy numbers based on centroid point and spread
}

\author{
Ahmad Syafadhli Abu Bakar* and Alexander Gegov \\ School of Computing, University of Portsmouth, United Kingdom
}

\begin{abstract}
Centroid and spread are commonly used approaches in ranking fuzzy numbers. Some experts rank fuzzy numbers using centroid or spread alone while others tend to integrate them together. Although a lot of methods for ranking fuzzy numbers that are related to both approaches have been presented, there are still limitations whereby the ranking obtained is inconsistent with human intuition. This paper proposes a novel method for ranking fuzzy numbers that integrates the centroid point and the spread approaches and overcomes the limitations and weaknesses of most existing methods. Proves and justifications with regard to the proposed ranking method are also presented.
\end{abstract}

Keywords: Consistent ranking, fuzzy numbers, centroid point, spread, human intuition

\section{Introduction}

Ranking fuzzy numbers plays an important role in decision making in fuzzy environment. Many ranking methods have been presented in the literature since this idea was first introduced by [1]. Among others were [2-8]. Basically, ranking fuzzy numbers provides the appropriate technique to deal with fuzzy numbers for decision making problems [9].

The literature on ranking fuzzy numbers classifies ranking methods into four categories. One of them is fuzzy mean and spread. In ranking fuzzy numbers, the mean is generally specified as the centroid of the fuzzy numbers. The concept of centroid in ranking fuzzy numbers was first introduced in [10] and this was later followed by $[11,12]$. However, the methods from [11, 12] have limitations as they only consider the positive sign for both numerator and denominator. The method from [11] produces similar ranking order for positive and negative fuzzy numbers while the method from [12] produces same ranking order for a mirror image situa-

\footnotetext{
${ }^{*}$ Corresponding author. Ahmad Syafadhli Abu Bakar, School of Computing, University of Portsmouth, PO1 3HE, United Kingdom. Tel.: +44 (0) 239284 6412; Fax: +44 (0) 239284 6411; E-mail: ahmad.abu-bakar@port.ac.uk.
}

tion of fuzzy numbers. It has been proved in [13] that the centroid formula given in $[11,12]$ does not satisfy the two properties of a correct centroid formula. Due to this [13] proposed a new centroid formula in ranking fuzzy numbers. Even though the method from [13] can be applied to various types of fuzzy numbers, this method is restricted to invertible fuzzy numbers only [14]. Therefore, a new centroid formula was proposed in [14] which is not only applicable to various types of fuzzy numbers but also satisfies the properties of a correct centroid formula. However, no clarification on ranking fuzzy numbers was introduced. Then $[15,16]$ proposed significant variations of the methods from [11, 12] but they produced inconsistent ranking order due to complexity. Later on [17] presented the same ranking method using the distance between the centroid but the methods produced ranking order such that the ordering is inconsistent with human intuitions and showed pitfalls in discriminating symmetrical fuzzy numbers of different spread.

Research on applicability of most ranking methods based on centroid to correctly rank fuzzy numbers is still ongoing but these methods cannot be used when embedded fuzzy numbers of different spread are considered. Due to this, several experts have proposed a 
combination of centroid and spread as a ranking fuzzy numbers method [18-20] were among the first that presented the ranking methods using both approaches. The method from [18] was unable to rank fuzzy numbers of different normality while the methods from $[19,20]$ could only be applied to trapezoidal fuzzy numbers [9, 21-23] later proposed some adjustments to previous ranking methods but all of them were inconsistent with human intuition.

To overcome the drawbacks mentioned above, this paper introduces a new ranking method which integrates centroid point and spread approaches for ranking fuzzy numbers. This paper is organised as follows. Preliminaries are given in Section 2. These are followed by discussions on shortcomings of existing ranking methods in Section 3. Section 4 covers the validation and proves of the proposed ranking method. Section 5 discusses the applicability of the proposed ranking method to other cases of fuzzy numbers by comparing the results obtained with the ones from other existing methods. Finally, a conclusion is drawn in Section 6.

\section{Theoretical preliminaries}

Based on [9], some basic concepts used in this paper are illustrated as follows.

\subsection{Trapezoidal fuzzy numbers}

A trapezoidal fuzzy number can be represented by the following membership function given by

$$
\begin{aligned}
& \mu_{A_{i}}(x) \\
& =\left(a_{i 1}, a_{i 2}, a_{i 3}, a_{i 4}\right) \\
& =\left\{\begin{array}{cc}
\frac{x-a_{i 1}}{a_{i 2}-a_{i 1}} & \text { if } a_{i 1} \leq x \leq a_{i 2} \\
1 & \text { if } a_{i 2} \leq x \leq a_{i 3} \\
\frac{a_{i 4}-x}{a_{i 4}-a_{i 3}} & \text { if } a_{i 3} \leq x \leq a_{i 4} \\
0 & \text { otherwise }
\end{array}\right.
\end{aligned}
$$

For a trapezoidal fuzzy number, if $a_{i 2}=a_{i 3}$, then the fuzzy number is in the form of a triangular fuzzy number. However, if $a_{i 1}=a_{i 2}=a_{i 3}=a_{i 4}$ for both triangular and trapezoidal fuzzy numbers, then both fuzzy numbers are said to be in the form of a singleton fuzzy number (crisp value). The length between $a_{i 1}$ and $a_{i 4}$ is known as the core of the fuzzy numbers.

\subsection{Generalized trapezoidal fuzzy numbers}

A fuzzy number $A=\left(a_{i 1}, a_{i 2}, a_{i 3}, a_{i 4} ; w_{A}\right)$ is called a generalized trapezoidal fuzzy number with $a_{i 1}, a_{i 2}, a_{i 3}, a_{i 4}$ are real numbers and $w_{A}$ represents the height of the fuzzy number $A$ such that $w_{A} \varepsilon[0,1]$. When $a_{i 2}=a_{i 3}, A$ is known as a generalized triangular fuzzy numbers [20].

\subsection{Standardized generalized trapezoidal fuzzy numbers}

If the fuzzy number $A$ has the property such that $1<a_{i 1}<a_{i 2}<a_{i 3}<a_{i 4}<1$, then $\tilde{A}$ is called a standardized generalized trapezoidal fuzzy number and is denoted as [9]

$$
\tilde{A}=\left(\tilde{a}_{i 1}, \tilde{a}_{i 2}, \tilde{a}_{i 3}, \tilde{a}_{i 4} ; w_{\tilde{A}}\right)
$$

Furthermore if $\tilde{a}_{i 2}=\tilde{a}_{i 3}$ then $\tilde{A}$ is known as a standardized generalized triangular fuzzy number. Any generalized fuzzy number may be transformed into a standardized generalized fuzzy number by normalization as described in (2).

$$
\begin{aligned}
\tilde{A} & =\left(\frac{a_{i 1}}{k}, \frac{a_{i 2}}{k}, \frac{a_{i 3}}{k}, \frac{a_{i 4}}{k} ; w_{A}\right) \\
& =\left(\tilde{a}_{i 1}, \tilde{a}_{i 2}, \tilde{a}_{i 3}, \tilde{a}_{i 4} ; w_{\tilde{A}}\right)
\end{aligned}
$$

where $k=\max \left(a_{i 1}, a_{i 2}, a_{i 3}, a_{i 4}\right)$.

It should be noted that in the normalization process only the components of fuzzy numbers are changed where $a_{i 1}, a_{i 2}, a_{i 3}, a_{i 4}$ are changed to $\tilde{a}_{i 1}, \tilde{a}_{i 2}, \tilde{a}_{i 3}, \tilde{a}_{i 4}$ but the height of the fuzzy number remains the same [9].

\section{Literature review}

Although numerous approaches for ranking fuzzy numbers have been proposed, there are still shortcomings demonstrated by the recently proposed methods in ranking fuzzy numbers consistently with human intuition. In this section, limitations of the existing ranking methods are discussed and analysed using three counter examples shown below. It should be noted that all fuzzy number examples used from this section onwards are in the form of standardized generalized fuzzy numbers.

Example 1 illustrates the limitations of $[9,22,24]$ in producing a consistent ranking order for the following cases with fuzzy numbers. 


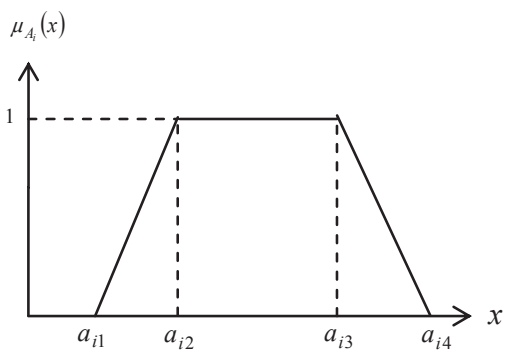

Fig. 1. Trapezoidal fuzzy number.

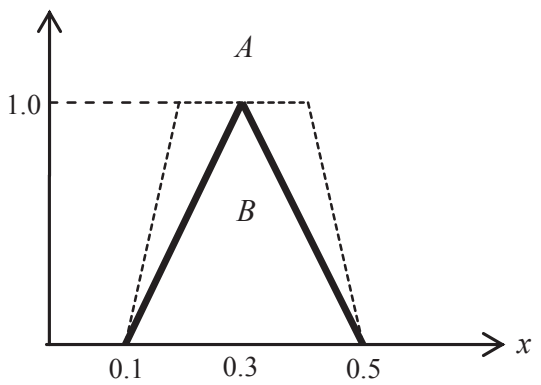

Fig. 2. Fuzzy numbers A and B of Example 1.

Example 1: Consider the following sets of fuzzy numbers adopted from $[9,22]$ and shown in Fig. 2.

$$
A=(0.1,0.2,0.4,0.5 ; 1.0), \quad B=(0.1,0.3,0.5 ; 1.0)
$$

Using [9] method, the ranking order of fuzzy numbers for this case is $B \succ A$, since the defuzzified value for both fuzzy numbers is the same, hence the spread value should be used as the discriminating factor. However, the result obtained by [9] is inconsistent with human intuition due to the centroid of $A$ is greater than $B$ which implies that $A$ should be intuitionally ranked higher than $B$ (i.e. $A \succ B$ ) $[17,22,24]$ on the other hand treated both fuzzy numbers as equal, $A \approx B$ which is unreasonable and deviate from human intuition. Therefore, it can be concluded that all the aforementioned ranking methods produce results which are inconsistent with human intuition particularly for Example 1.

Example 2 analyses the illogical ranking order of another case with fuzzy numbers obtained by [17].

Example 2: Consider the following sets of fuzzy numbers adopted from [25] shown in Fig. 3.

$$
A=(0.2,0.5,0.8 ; 1.0), \quad B=(0.4,0.5,0.6 ; 1.0)
$$

Although [17] method has solved the problem faced by $[9,22]$, this method has shortcomings when applied to fuzzy numbers in Example 2. Using their method,

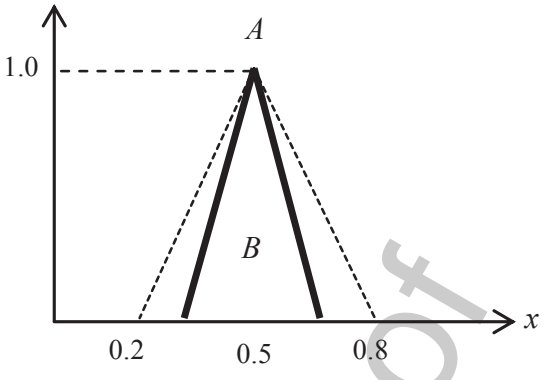

Fig. 3. Fuzzy numbers A and B of Example 2.

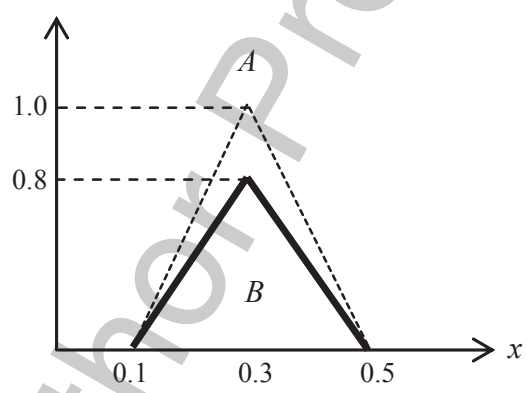

Fig. 4. Fuzzy numbers $A$ and $B$ of Example 3 .

the ranking order obtained is equal ranking $(A \approx B)$ because the distance between the centroid for both fuzzy numbers is the same. Thus, [17] method produces unreasonable ranking order for this case with fuzzy numbers.

Example 3: Consider the following sets of fuzzy numbers adopted from [22] shown in Fig. 4.

$$
A=(0.1,0.3,0.5 ; 1.0), \quad B=(0.1,0.3,0.5 ; 0.8)
$$

Since $w_{A}>w_{B}$, the centroid point for fuzzy number $A$ is greater than $B$. Therefore, it is obvious that the ranking order of fuzzy numbers which is consistent with human intuition for this example should be $A \succ B$. However, the application of the method from [25] to this example produced different ranking order for different degrees of optimism. Therefore, the method from [25] had pitfall in ranking fuzzy numbers for this example by giving ranking order that is unreasonable and inconsistent with human intuition.

\section{Research methodology}

To overcome the limitations of existing methods, this study introduces a novel hybrid methodology for ranking fuzzy numbers method based on centroid point and spread (CPS). The centroid point is utilised in CPS 
due to the effectiveness of this approach in ranking various cases of fuzzy numbers which are suited to human intuition. The spread method, on the other hand, is integrated with the centroid point to cater for the pitfalls faced by the existing ranking methods, as already discussed in Section 3. The full illustration of the proposed new ranking method is presented below.

Since centroid is considered as the main factor in ranking fuzzy numbers by human intuition [17], the centroid method from [14] is used here as one of the components of CPS. This is due to the fact that this centroid method has the ability to deal with numerous types of fuzzy numbers as discussed in [17]. Therefore, the centroid method from [14] is proposed here as one of the components in the CPS ranking method which is defined as follows.

Assume that a fuzzy number $A$ is generally described as $A=\left(a_{1}, a_{2}, a_{3}, a_{4} ; w_{A}\right)$, the horizontal $-x$ centroid equation of fuzzy number $A, x_{A}$ is calculated as

$$
x_{A}=\frac{\int_{-\infty}^{\infty} x f(x) d x}{\int_{-\infty}^{\infty} f(x) d x}
$$

and the vertical $-y$ centroid equation of fuzzy number $A, y_{A}$ is calculated as

$$
y_{A}=\frac{\int_{0}^{w_{A_{i}}} \alpha\left|A_{i}^{\alpha}\right| d \alpha}{\int_{0}^{w_{A_{i}}}\left|A_{i}^{\alpha}\right| d \alpha}
$$

where

$\left|A_{i}^{\alpha}\right|$ is the length of the $\alpha$ - cuts of fuzzy number $A$, $x_{A} \in[-1,1]$ and $y_{A} \in\left[0, w_{A}\right]$.

As discussed in Section 3, there are some cases where the centroid method is unable to rank the fuzzy numbers appropriately, especially when fuzzy numbers of different spread are considered. Therefore, considering spread in the formulation is important.

\subsection{Spread in ranking fuzzy numbers and decision making}

The roles play by the spread can be in twofold. They are

\section{Capability in Ranking Fuzzy Numbers.}

Although, centroid point can rank almost all cases of fuzzy numbers, spread does gives great assistance when centroid point fails to rank the following fuzzy numbers cases
a. Fuzzy numbers of different spreads.
b. Embedded fuzzy numbers.

\section{Role in Decision Making}

In decision making environment, the decision makers can be categorised into three namely pessimistic, neutral and optimistic $[5,27]$. This implies that they have different views in terms of the spread of fuzzy numbers, although the fuzzy numbers they observe are of the same situation. Therefore, the ranking order might be differ from one to another which indicates that spread is also important in the decision making process.

Thus, it is crucial not only to consider centroid point but also spread in ranking fuzzy numbers and decision making applications.

\subsection{Spread formula for fuzzy numbers}

According to [9], the spread is not considered as important as the centroid in ranking fuzzy numbers. However, the spread does provide great assistance to the centroid when dealing with fuzzy numbers in certain cases such as the ones presented in Section 3. Therefore, a new spread formula is proposed here based on the distance from the centroid point.

The distance along the $x$ - axis from the centroid of $x$ - value is defined as

$$
\begin{aligned}
i_{A}=\operatorname{dist}\left(a_{4}-a_{1}\right) & =\left|a_{4}-x_{A}\right|+\left|x_{A}-a_{1}\right| \\
& =\left|a_{4}-a_{1}\right|
\end{aligned}
$$

Further on, the distance along the vertical $y$ - axis from the centroid of $y$ - value is defined as

$$
i i_{A}=y_{A}
$$

Therefore, spread of $A, s(A)$ is defined as

$$
s(A)=i_{A} \times i i_{A}
$$

where $i$ and $i i$ are $\operatorname{dist}\left(a_{4}-a_{1}\right)$ and $y_{A}$ respectively.

$$
s(A), i_{A}, i i_{A}, \operatorname{dist}\left(a_{4}-a_{1}\right) \in[0,1] .
$$

The following figure is the illustration of the proposed spread methodology.

\subsection{Properties of spread method}

The relevant properties considered for justifying the spread in ranking fuzzy numbers depend on the usefulness within the domain of research and the list of these properties can be extended further. The applicability of the proposed spread method in ranking fuzzy numbers is illustrated using the following properties. 
Let $A$ and $B$ be trapezoidal and triangular generalized fuzzy numbers respectively.

Property 1: If $A$ and $B$ are embedded and having similar core, then $s(A)>s(B)$.

Proof: Since $A$ and $B$ are embedded and having similar core, hence we know that

$$
x_{A}=x_{B} \text { and } y_{A}>y_{B} .
$$

Then, from equation (1) we have $i_{A}=i_{B}$ and $i i_{A}>$ $i i_{B}$.

Therefore, $s(A)>s(B)$.

Figure 1 is the best representation of this property.

Property 2: If $A$ is a vertical fuzzy numbers, then $s(A)=0$.

Proof: For any crisp (real) numbers, we know that $a_{1}=$ $a_{2}=a_{3}=a_{4}$ implies that $i_{A}=0$ and $i i_{A}=w / 3$.

Therefore, $s(A)=0$.

Property 3: If $A$ is an asymmetrical triangular fuzzy numbers then $s(A)=i_{A} \times i i_{A}$.

Proof: For any asymmetrical triangular fuzzy numbers, it is obvious that $a_{2}=a_{3} \neq x_{A}$.

Then, by definition, we have $\operatorname{dist}\left(a_{4}-a_{3}\right)+$ $\operatorname{dist}\left(a_{3}-a_{1}\right)=\operatorname{dist}\left(a_{4}-a_{1}\right)=i_{A}$.

Therefore, $s(A)=i_{A} \times i i_{A}$.

Therefore, the proposed ranking fuzzy numbers is defined as follows.

Definition 4. The CPS ranking index value is defined as

$$
C P S(A)=x_{A}^{*} \times y_{A}^{*} \times\left(1-s_{A}\right)
$$

where

$x_{A}^{*}$ is the horizontal $-x$ centroid for generalized fuzzy number $A$

$y_{A}^{*}$ is the horizontal $-y$ centroid for generalized fuzzy number $A$

$s_{A}$ is the spread for fuzzy number $A$

$C P S(A) \in[-1,1]$

If $C P S(A)>C P S(B)$, then $A \succ B$. (i.e. $A$ is ranked higher than $B)$.

If $C P S(A)<C P S(B)$, then $A \prec B$. (i.e. $A$ is ranked lower than $B)$.

If $C P S(A)=C P S(B)$, then $A \approx B$. (i.e. the ranking for $A$ and $B$ is equal).

\section{Comparative analysis}

In this section, the CPS method is compared with other existing methods in ranking fuzzy numbers. The CPS method demonstrates its reliability for ranking the fuzzy numbers from Section 3 and also stamps its supremacy on several other examples of fuzzy numbers. This comparative analysis is important to ensure that the CPS method can handle any cases of fuzzy numbers in the same way as other existing methods. Consistent ranking result means the ranking order of fuzzy numbers obtained is correct and consistent with human intuition. Without loss of generality, cases of fuzzy numbers examined in $[9,22]$ are illustrated as follows.

\subsection{Case 1}

Consider the two fuzzy numbers $A$ and $B$ shown in Fig. 2. The correct ranking order of fuzzy numbers for this case should be $A \succ B$ due to the fact that the centroid of fuzzy number $A$ is greater than fuzzy number $B$ [17]. Based on Table 1 [9] produced unreasonable ranking order that is inconsistent with human intuition $(B \succ A)$ since they treated fuzzy number with smaller centroid as greater than the other. The attempt of [22] to overcome the limitations of the method from [9] results in an inconsistent ranking order in which the method treated both fuzzy numbers as equal $(A \approx B)$. The same ranking order is also obtained using the methods from $[11,12,24]$. This outcome implies that these methods are unable to differentiate between fuzzy numbers appropriately. Using the CPS ranking method, the ranking order produced is consistent with the method [17]. The latter produces a ranking order that is consistent with human intuition by placing the fuzzy number with higher centroid, higher in the ranking order.

Table 1

\begin{tabular}{|c|c|c|c|c|}
\hline \multirow[t]{2}{*}{ Method } & \multicolumn{2}{|c|}{ Fuzzy numbers } & \multirow[t]{2}{*}{ Ranking results } & \multirow[t]{2}{*}{ Evaluation } \\
\hline & $A$ & $B$ & & \\
\hline$[11]$ & 0.583 & 0.583 & $A \approx B$ & Inconsistent \\
\hline [12] & 0.150 & 0.150 & $A \approx B$ & Inconsistent \\
\hline [9] & 0.254 & 0.258 & $A \prec B$ & Inconsistent \\
\hline [24] & 0.300 & 0.300 & $A \approx B$ & Inconsistent \\
\hline [22] & 0.300 & 0.300 & $A \approx B$ & Inconsistent \\
\hline$[17]$ & 0.333 & 0.222 & $A \succ B$ & Consistent \\
\hline CPS & 0.103 & 0.077 & $A \succ B$ & Consistent \\
\hline
\end{tabular}

Comparative results of case 1 
Table 2

Comparative results of case 2

\begin{tabular}{|c|c|c|c|c|}
\hline \multirow[t]{2}{*}{ Method } & \multicolumn{2}{|c|}{ Fuzzy numbers } & \multirow[t]{2}{*}{ Ranking results } & \multirow[t]{2}{*}{ Evaluation } \\
\hline & $A$ & $B$ & & \\
\hline [11] & 0.583 & 0.583 & $A \approx B$ & Inconsistent \\
\hline [12] & 0.150 & 0.150 & $A \approx B$ & Inconsistent \\
\hline [9] & 0.258 & 0.278 & $A \prec B$ & Inconsistent \\
\hline [24] & 0.500 & 0.500 & $A \approx B$ & Inconsistent \\
\hline$[22]$ & 0.300 & 0.300 & $A \approx B$ & Inconsistent \\
\hline [26] & 0.240 & 0.240 & $A \approx B$ & Inconsistent \\
\hline [17] & 0.111 & 0.111 & $A \approx B$ & Inconsistent \\
\hline \multirow[t]{3}{*}{ [25] } & 1.000 & 1.000 & $A \prec B$ & $\begin{array}{l}\text { Consistent / } \\
\text { Inconsistent } \\
\text { depending on } \alpha\end{array}$ \\
\hline & 1.000 & 1.000 & $A \approx B$ & \\
\hline & 1.000 & 1.000 & $A \succ B$ & \\
\hline CPS & 0.103 & 0.077 & $A \succ B$ & Consistent \\
\hline
\end{tabular}

\subsection{Case 2}

Consider the two fuzzy numbers $A$ and $B$ shown in Fig. 3. The correct ranking order of fuzzy numbers for this case should be $B \succ A$. This is in accordance with $[12,18]$ where it was pointed out that the ranking order for a fuzzy number with a lower spread value is greater than the other provided that the centroid value of fuzzy numbers under consideration is the same. It can be seen from Table 2 that the ranking methods from $[11,12$, $17,24,26]$ are unable to differentiate between fuzzy numbers whereby they produce equal ranking $(A \approx B)$ for this case. The ranking method from [25], on the other hand, captures the actual preference of decision makers by utilising the degree of optimism in obtaining the ranking order for the fuzzy numbers. The CPS method produces consistent ordering in line with [9] and [22] that rank the fuzzy numbers correctly by giving priority to fuzzy numbers with lower spread which is in line with human intuition. It can also be seen that most of the presented ranking methods are unable to solve this case of fuzzy numbers.

\subsection{Case 3}

Consider the two fuzzy numbers $A$ and $B$ shown in Fig. 4. As mentioned in Section 3, the method from [25] was unable to give appropriate ranking order for the fuzzy numbers in Fig. 4. It should be noted that the ranking values obtained by the method from [25] were the same but the ranking results were different because the method gave different ranking for different levels of degree of optimism. Obviously, without considering the degree of optimism, a ranking method should rank
Table 3

Comparative results of case 3

\begin{tabular}{lcccc}
\hline Method & \multicolumn{2}{c}{ Fuzzy numbers } & Ranking results & Evaluation \\
\cline { 2 - 3 } & $A$ & $B$ & & \\
\hline$[11]$ & 0.583 & 0.461 & $A \succ B$ & Consistent \\
{$[12]$} & 0.150 & 0.120 & $A \succ B$ & Consistent \\
{$[9]$} & 0.258 & 0.206 & $A \succ B$ & Consistent \\
{$[24]$} & 0.240 & 0.240 & $A \approx B$ & Inconsistent \\
{$[22]$} & 0.300 & 0.282 & $A \succ B$ & Consistent \\
{$[26]$} & 0.150 & 0.133 & $A \succ B$ & Consistent \\
{$[17]$} & 0.244 & 0.196 & $A \succ B$ & Consistent \\
{$[25]$} & 1.000 & 1.000 & $A \approx B$ & Inconsistent \\
& 1.000 & 1.000 & $A \approx B$ & \\
& 1.000 & 1.000 & $A \approx B$ & \\
CPS & 0.077 & 0.062 & $A \succ B$ & Consistent \\
\hline & & & &
\end{tabular}

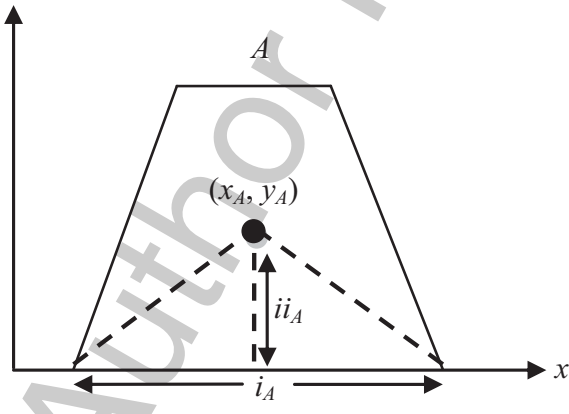

Fig. 5. Component of spread, $i_{A}$ and $i i_{A}$ and the centroid point, $\left(x_{A}\right.$, $\left.y_{A}\right)$.

$A \succ B$ due to level of confidence of decision makers that fuzzy number $A$ is greater than $B$. In Table 3 , it was also shown that [24] produced inconsistent ranking order by treating both fuzzy numbers as equal. Using the CPS method, consistent ranking order is obtained in line with $[9,11,12,22,26]$ where a fuzzy number with greater height is ranked higher than a fuzzy number with lower height.

\subsection{Case 4}

Consider the reflection case of the two non-overlapping fuzzy numbers $A$ and $B$ shown in Fig. 6 and Table 4 as comparative results. It is obvious that fuzzy number $B$ is situated on the farthest right compared to fuzzy number $A$. Therefore, the ranking order that is consistent with human intuitions should be $B \succ A$. The methods from [11, 24] were unable to differentiate between these fuzzy numbers, hence producing an inconsistent ranking order. However, when using the CPS method, the ranking order obtained is in line with $[9,12,17,22,26]$ where the ranking order is consistent with human intuition.
335 


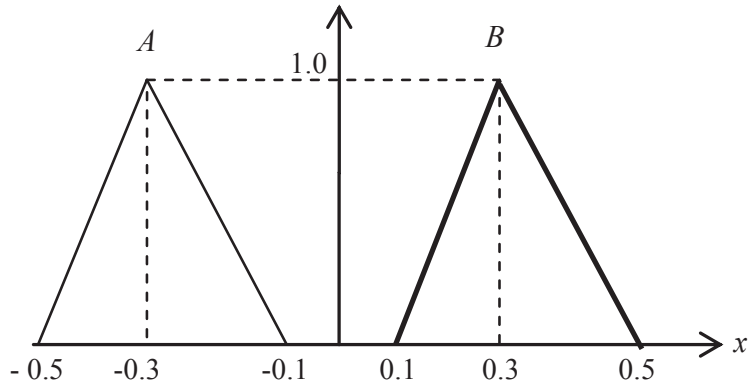

Fig. 6. Fuzzy Numbers $A$ and $B$ of Example 4.

Table 4

Comparative results of case 4

\begin{tabular}{|c|c|c|c|c|}
\hline \multirow[t]{2}{*}{ Method } & \multicolumn{2}{|c|}{ Fuzzy numbers } & \multirow[t]{2}{*}{ Ranking results } & \multirow[t]{2}{*}{ Evaluation } \\
\hline & $A$ & $B$ & & \\
\hline [11] & 0.583 & 0.583 & $A \approx B$ & Consistent \\
\hline$[12]$ & -0.150 & 0.150 & $A \prec B$ & Consistent \\
\hline [9] & -0.258 & 0.258 & $A \prec B$ & Consistent \\
\hline [24] & 0 & 0 & $A \approx B$ & Inconsistent \\
\hline [22] & -0.300 & 0.300 & $A \prec B$ & Consistent \\
\hline [26] & 0.150 & 0.133 & $A \succ B$ & Consistent \\
\hline [17] & 0 & 0.600 & $A \prec B$ & Consistent \\
\hline CPS & -0.077 & 0.077 & $A \prec B$ & Consistent \\
\hline
\end{tabular}

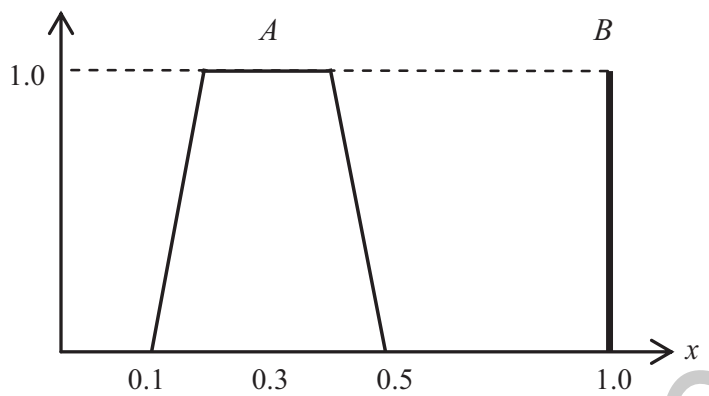

Fig. 7. Fuzzy Numbers $A$ and $B$ of Example 5.

Table 5

Comparative results of case 54

\begin{tabular}{|c|c|c|c|c|}
\hline \multirow[t]{2}{*}{ Method } & \multicolumn{2}{|c|}{ Fuzzy numbers } & \multirow[t]{2}{*}{ Ranking results } & \multirow[t]{2}{*}{ Evaluation } \\
\hline & $A$ & $B$ & & \\
\hline [11] & $\mathrm{x}$ & $X$ & - & Inconsistent \\
\hline [12] & $\mathrm{x}$ & $\mathrm{X}$ & - & Inconsistent \\
\hline [9] & 0.254 & 0.258 & $A \prec B$ & Consistent \\
\hline [24] & $\mathrm{x}$ & $\mathrm{X}$ & & Inconsistent \\
\hline$[22]$ & 0.300 & 1 & $A<B$ & Inconsistent \\
\hline [26] & $\mathrm{x}$ & $\mathrm{X}$ & - & Inconsistent \\
\hline [17] & 0.333 & 1.082 & $A \prec B$ & Consistent \\
\hline CPS & 0.077 & 0.333 & $A \prec B$ & Consistent \\
\hline
\end{tabular}

Note: ' $\mathrm{x}$ ' the ranking method as unable to rank the fuzzy numbers. '-' not applicable for the ranking method.

It is understandable that each presented method of ranking fuzzy numbers has its own strengths and weaknesses. Based on the analysis above, there are some methods that can deal with cases of fuzzy numbers proposed by $[9,22]$ effectively while some produce irrelevant results for certain cases. Nevertheless, in each case examined above, the CPS method is more likely to produce consistent ranking results for all cases with fuzzy numbers. This implies that the CPS method can deal with each case of fuzzy numbers proposed by [9, 22] effectively.

\section{Conclusion}

This study proposes a novel method for ranking fuzzy numbers based on centroid point and spread. The method utilises the centroid point formula due to its applicability to all types of fuzzy numbers. At the same time, a novel spread approach is introduced to overcome the weaknesses of most existing methods in calculating the spread of fuzzy numbers. It is shown that the CPS method not only produces correct ranking order for each case with fuzzy numbers considered but also overcomes the limitations of most existing methods in ranking fuzzy numbers by producing a ranking order that is consistent with human intuition. In conclusion, the proposed method possesses intuitional concepts for ranking fuzzy numbers as well as for decision making analysis. Therefore, it is expected that this method can be further improved and validated for decision making problems.

\section{References}

[1] R. Jain, Decision-making in the presence of fuzzy variable, IEEE Transactions on Man and Cybernetic 6 (1976), 698-703.

[2] P. Fortemps and M. Roubens, Ranking and defuzzification methods based on area compensation, Fuzzy Sets and Systems 82 (1996), 319-330. tent ran
values. 
[3] V.V. Cross and M. Setnes, A generalized model for ranking fuzzy sets, IEEE World Congress on Computational Intelligence 1 (1998), 773-778.

[4] H.L. Kwang and J.H. Lee, A method for ranking fuzzy numbers and its application to decision-making, IEEE Transactions on Fuzzy Systems 7 (1999), 677-685.

[5] H.S. Lee, A new fuzzy ranking method based on fuzzy preference relation, Systems, Man, and Cybernetics 5 (2000), 3416-3420.

[6] G. Facchinetti, Ranking function induced by weighted average of fuzzy numbers, Fuzzy Optimization and Decision Making 1(3) (2002), 313-327.

[7] A. Asady and A. Zendehnam, Ranking fuzzy numbers by distance minimization, Applied Mathematical Modeling 31 (2007), 2589-2598.

[8] S.M. Chen and J.H. Chen, Fuzzy risk analysis based on ranking generalized fuzzy numbers with different heights and different spreads, Expert Systems with Applications 36 (2009), 6833-6842.

[9] D. Mohamad, N.H. Sulaiman and A.S.A. Bakar, Solving industrial decision making using fuzzy numbers, IEEE Symposium on Business, Engineering and Industrial Applications (2011), 217-274.

[10] R.R. Yager, A procedure for ordering fuzzy subsets of the unit interval, Information Sciences 24 (1981), 143-161.

[11] C.H. Cheng, A new approach for ranking fuzzy numbers by distance method, Fuzzy Sets and System 95 (1998), 307-317.

[12] C.T. Chu and C.T. Tsao, Ranking fuzzy numbers with an area between the centroid point and original point, Computer and Mathematics with Applications 43 (2002), 111-117.

[13] Y.M. Wang, J.B. Yang, D.L. Xu and K.S. Chin, On the centroids of fuzzy numbers, Fuzzy Sets and Systems 157 (2006), 919-926.

[14] B.S. Shieh, An approach to centroids of fuzzy numbers, International Journal of Fuzzy Systems 9 (2007), 51-54.

[15] Y.J. Wang and H.S. Lee, The revised method of ranking fuzzy numbers with an area between the centroid and original points, Computers and Mathematics with Applications 55(9) (2008), 2033-2042.
[16] C.L. Xu and L.L. Wei, An improved method for ranking fuzzy numbers based on the centroids, Seventh International Conference on Fuzzy System and Knowledge Discovery (2010), 442-446.

[17] L.Q. Dat, V.F. Yu and S.Y. Chou, An improved ranking method for fuzzy numbers based on the centroid index, International Journal of Fuzzy Systems 14(3) (2012), 413-419.

[18] L.H. Chen and H.W. Lu, The preference order of fuzzy numbers, Computers and Mathematics with Applications $\mathbf{4 4}$ (2002), 1455-1465.

[19] S.J. Chen and S.M. Chen, A new method for handling multicriteria fuzzy decision making problems using FN-IOWA Operators, Cybernatics and Systems 34 (2003), 109-137.

[20] C.J. Chen and S.M. Chen, Fuzzy risk analysis based on the ranking of generalized trapezoidal fuzzy numbers, Applied Intelligence 26(1) (2007), 1-11.

[21] A.S.A. Bakar, D. Mohamad and N.H. Sulaiman, Ranking fuzzy numbers using similarity measure with centroid, IEEE International Conference on Science and Social Research (2010), 58-63.

[22] S.M. Chen and K. Sanguansat, Analyzing fuzzy risk based on a new fuzzy ranking method between generalized fuzzy numbers, Expert System with Applications 38 (2011), 2163-2171.

[23] A.S.A. Bakar, D. Mohamad and N.H. Sulaiman, Distance based ranking fuzzy numbers, Advances in Computational Mathematics and Its Applications 1(3) (2012), 146-150.

[24] A. Kumar, P. Singh, P. Kaur and A. Kaur, A new approach for ranking generalized trapezoidal fuzzy numbers, World Academy of Science, Engineering and Technology 68 (2010), 229-302.

[25] V.F. Yu, H.T.X. Chi and C.W. Shen, Ranking fuzzy numbers based on epsilon - deviation degree, Applied Soft Computing 13(8) (2013), 3621-3627.

[26] T. Allahviranloo and R. Saneifard, Defuzzification method for ranking fuzzy numbers based on center of gravity, Iranian Journal of Fuzzy Systems 9(6) (2012), 57-67.

[27] N. Ramli and D. Mohamad, On the jaccard index of similarity measure in ranking fuzzy numbers, MATEMATIKA 25(2) (2009), 157-165. 